\title{
Pharmacokinetic Concentration Time Point Reference
}

National Cancer Institute

\section{Source}

National Cancer Institute. Pharmacokinetic Concentration Time Point Reference. NCI

Thesaurus. Code C87976.

The point in time that acts as a fixed reference point to a pharmacokinetic concentration assessment. 